\title{
A Study Of Correlation Between Health Benefits \& Food Choices: A Case Study Using Raw \& Ripe Papaya
}

\author{
*Dr. Jyoti D. Vora, **Ms. Sneha R. Pednekar*Guide \\ Head, Department of Biochemistry \& Food Science and Quality ControlRamnarain Ruia College, Matunga, \\ Mumbai - 400019 \\ Department of Biochemistry \& Food Science and Quality ControlRamnarain Ruia College, Matunga, Mumbai- \\ 400019
}

\section{INTRODUCTION -}

Diet provides the necessary building blocks for our body to produce energy and to maintain good health. A balanced diet, therefore, is the foundation of good health. Clearly, food does much more than meet a basic physical need. It meets emotional, social, and psychological needs as well. To insure mental and emotional well-being, the diet must supply all the nutrients crucial for the proper growth and development of body both physiologically and emotionally. The food choices can have a significant impact on body functions. The deficiencies can impair life processes such as wound healing and metabolism. Likewise, some choices can increase the risk of disease. Hence, the concern about food choices that may have adverse effects on health is currently at the forefront of public health. Eating behaviour is a complex process and an understanding of the impact of the factors that affect food choice is vital given the priority for population dietary change. Healthy eating habits promote growth and reduce many risks associated with both immediate and long-term health problems. Thus, there is a need for a greater understanding of the determinants that affect food choice.

The key driver for eating is hunger but what we choose to eat is not determined solely by physiological or nutritional needs. Some of the other factors that influence food choice include:

1) Biological determinants such as hunger, appetite, satiety and taste

2) Economic determinants such as cost, income, availability

3) Physical determinants such as access, education, skills (e.g. cooking) \& time

4) Social determinants such as culture, religious beliefs, family, peers and meal patterns

5) Psychological determinants such as mood, stress and guilt

6) Attitudes, beliefs and knowledge about food

'Taste' is consistently reported as a major influence on food behaviour. In reality 'taste' is the sum of all sensory stimulation that is produced by the ingestion of a food. This includes taste, smell, appearance and texture of food. These sensory aspects are thought to influence, in particular, spontaneous food choice.

Diets high in fruits are widely recommended for their health-promoting properties. Carica papaya is a nutraceutical plant rich in vitamins, minerals, and phytochemicals, especially antioxidants. Health benefit properties of Carica papaya are widely recognized and have been scientifically documented since time immemorial. Therefore, the present study aims to create awareness about the consumption and the health benefits of papaya.

\section{REVIEW OF LITERATURE -}

From an early age, taste and familiarity influence behaviour towards food. A liking for sweetness and a dislike for bitterness are considered innate human traits, present from birth (Steiner 1977). Taste preferences and food aversions develop through experiences and are influenced by our attitudes, beliefs and expectations (Clarke 1998).There is little doubt that the cost of food is a primary determinant of food choice. Whether cost is prohibitive depends fundamentally on a person's income and socio-economic status. Low-income groups have a greater tendency to consume unbalanced diets and in particular have low intakes of fruit and vegetables (De Irala-Estevez et al. 2000). Accessibility to sale outlets is another important physical factor influencing food choice, which is dependent on resources such as transport and geographical location. Studies indicate that the level of education can influence dietary behaviour during adulthood (Kearney et al. 2000). With the majority of adult women and men in employment, the influence of work on health behaviours such as food choices is an important area of investigation (Devine 2003). Lack of time is frequently mentioned for not following nutritional advice, particularly by the young and well educated (Lappalainen et al. 1997). People living alone or cooking for one seek out convenience foods rather than cooking from basic ingredients. The influence of stress on food choice is complex and depends on the individual, the stressor and the circumstances. Interestingly, it appears that the influence of food on mood is related in part to attitudes towards particular foods. 
Education on how to increase fruit and vegetable consumption in an affordable way such that no further expense, in money or effort, is incurred has been proposed as a solution (Dibsdall et al. 2003). Efforts of governments, public health authorities, producers and retailers to promote fruit and vegetable dishes as value for money could also make a positive contribution to dietary change (Cox et al 1998b).

III. MATERIALS \& METHODS -

A carefully structured questionnaire was administered to the urban Indian subjects in the age group of $18-25$ years with the view of obtaining a holistic picture of their perspective towards their food choice of consumption of papaya. The questionnaire comprised of a series of questions regarding inclusion of papaya in the diet, its health benefits and general awareness. A total of 56 subjects were studied. The findings were statistically analyzed.

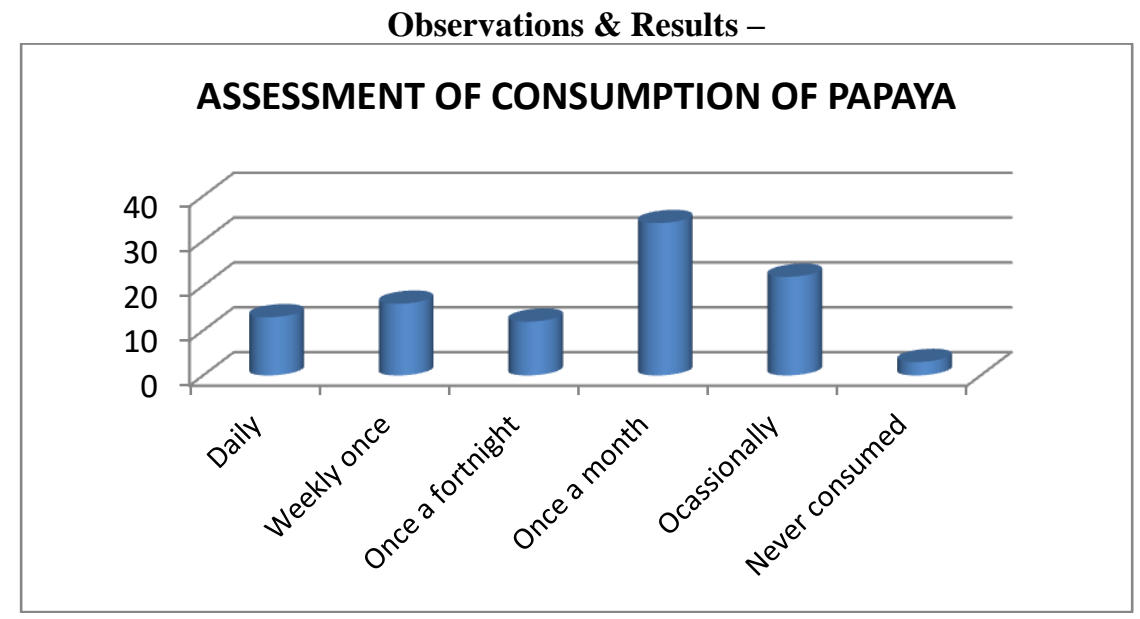

It is observed that only 3\% of the subjects under study had never consumed papaya. Their reasons for not consuming papaya was unappealing taste and smell.

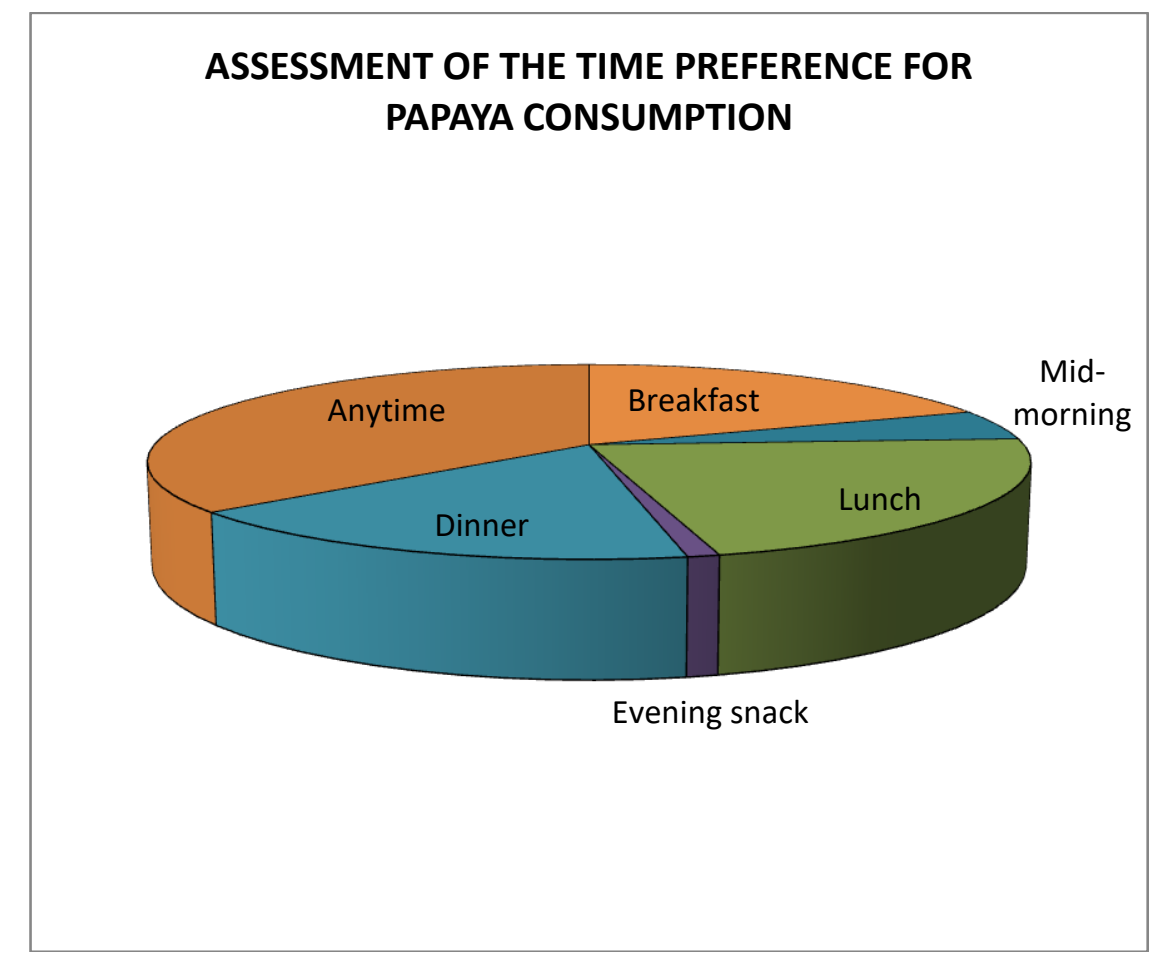

The consumption of papaya was less preferred in mid-morning and as evening snack. 


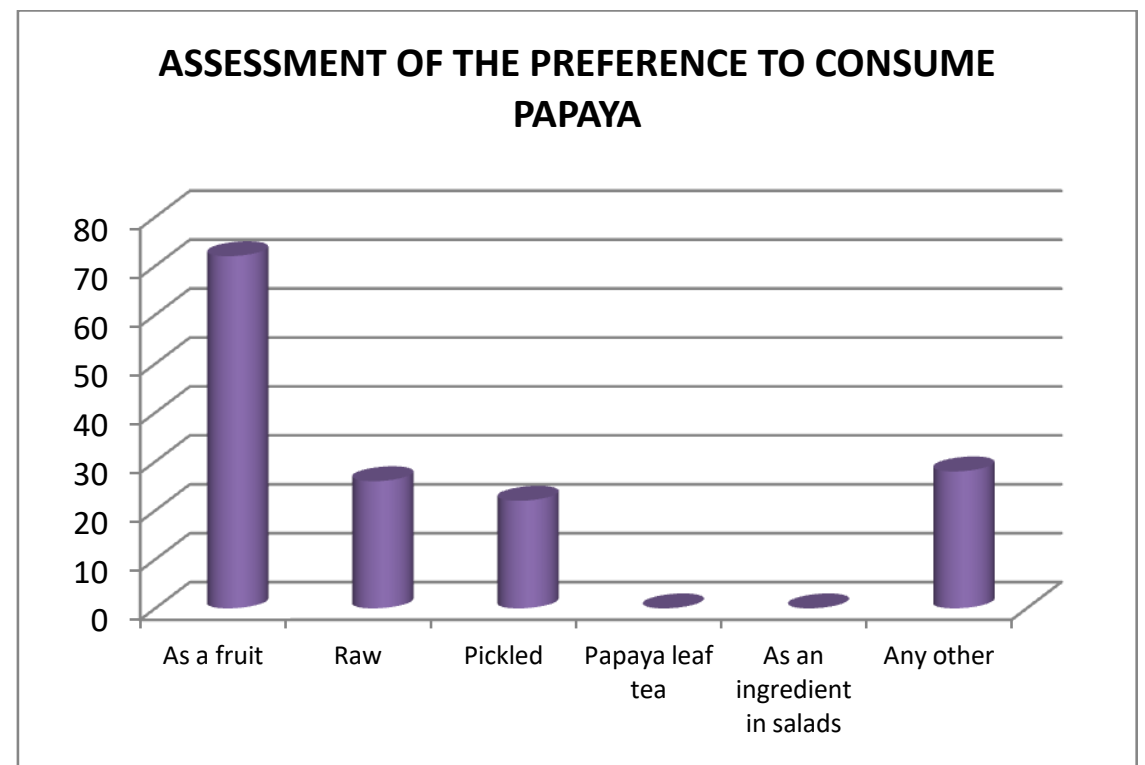

It was observed that none of the subjects under study had consumed papaya leaf tea or had included it as an ingredient of salad. However, $28 \%$ subjects also preferred consuming papaya in the form of curry.

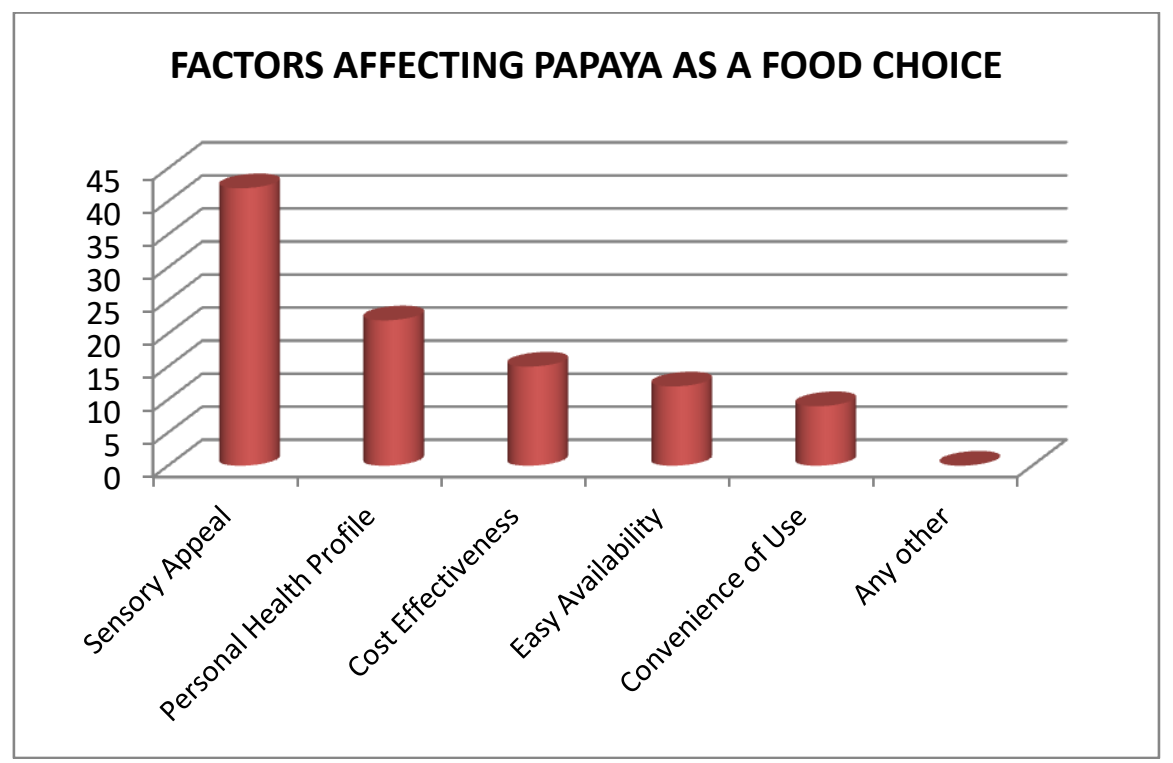

The taste and smell did not appeal to $42 \%$ of the subjects under study. However, certain subjects preferred not to consume papaya due to onset of diarrhoea-like symptoms.

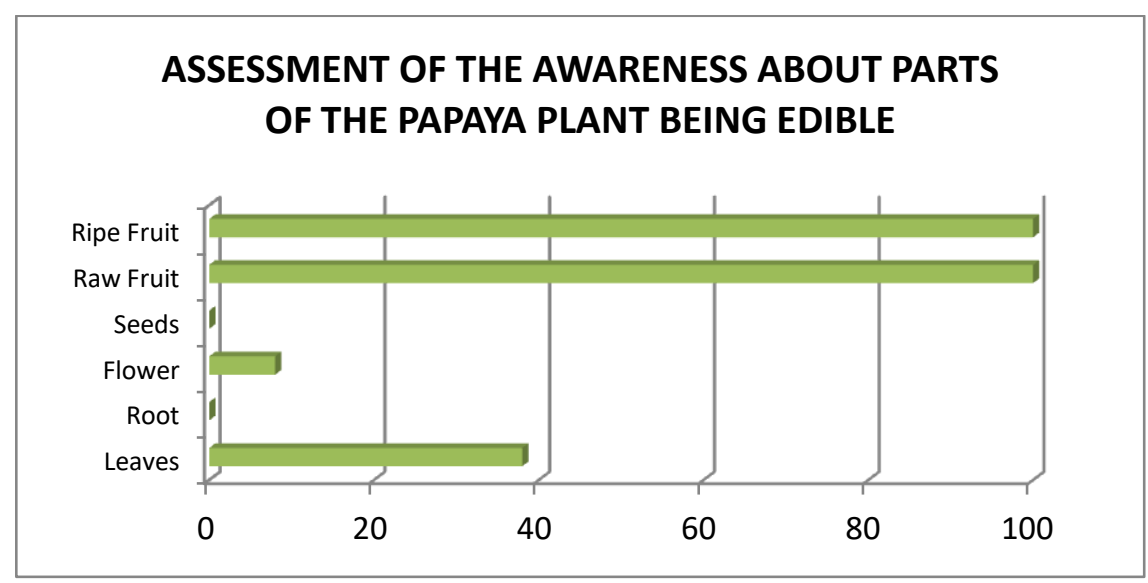

It was observed that none of the subjects were aware that the papaya seeds are edible. Most of the subjects were of the belief that squeezing papaya seeds causes night blindness. 
It was observed that $48 \%$ of the subjects under study were of the opinion that incorporation of papaya into the diet more frequently has various health benefits.

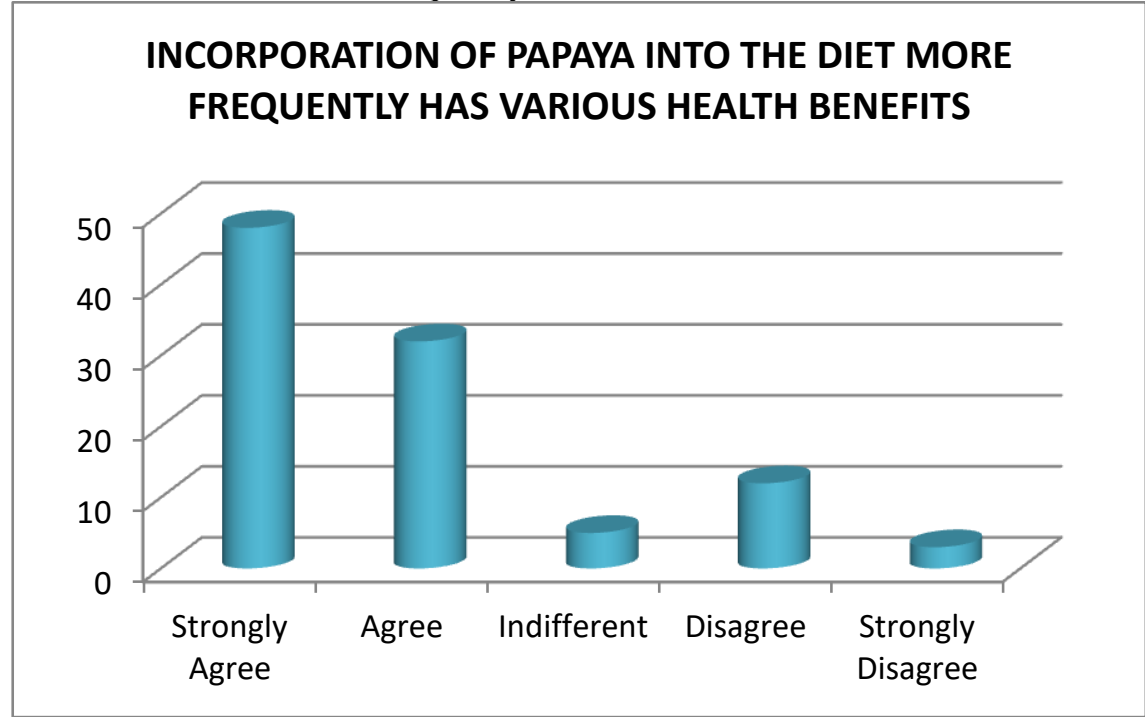

\section{ASSESSMENT OF THE AWARENESS OF HEALTH BENEFITS OF PAPAYA}

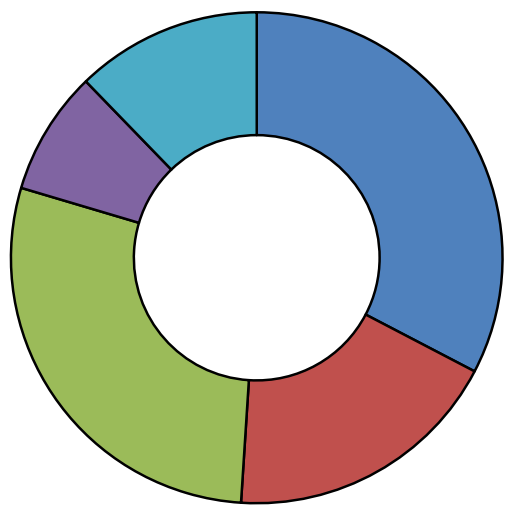

$\square$ Aids in digestion

$\square$ Diabetes control

$\square$ Reduce risk of heart diseases

$\square$ Management of gluten allergy

口Improves wound healing

$\square$ Any other

Most of the subjects under study were aware that papaya aids in digestion and is useful in reducing risk of heart diseases.

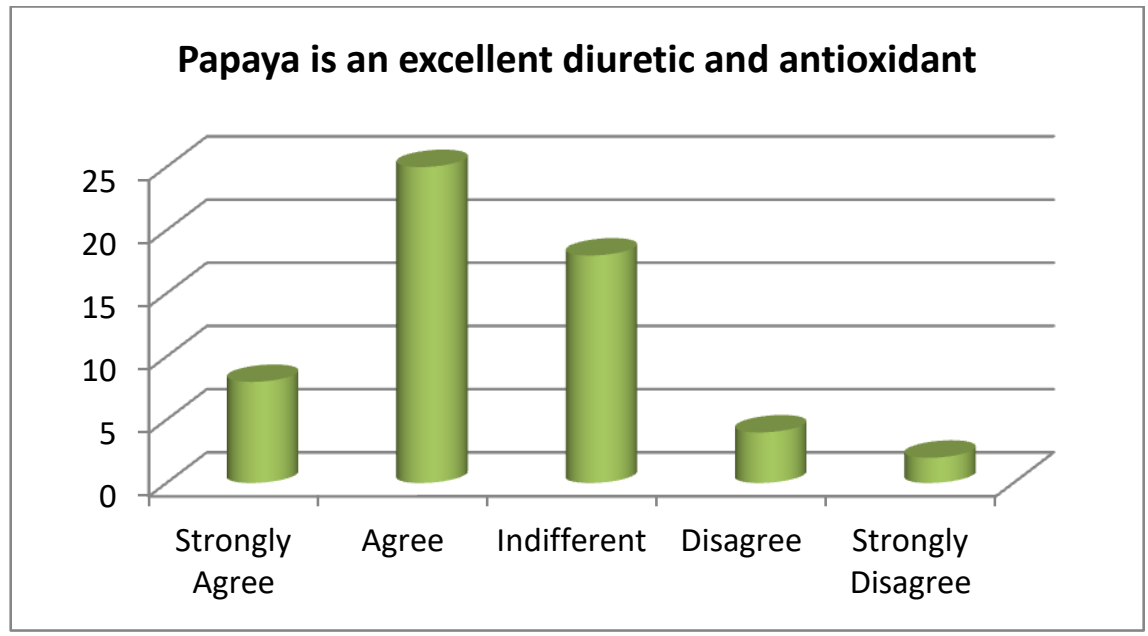

Most of the subjects under study were unaware of papaya being an excellent diuretic and antioxidant. 


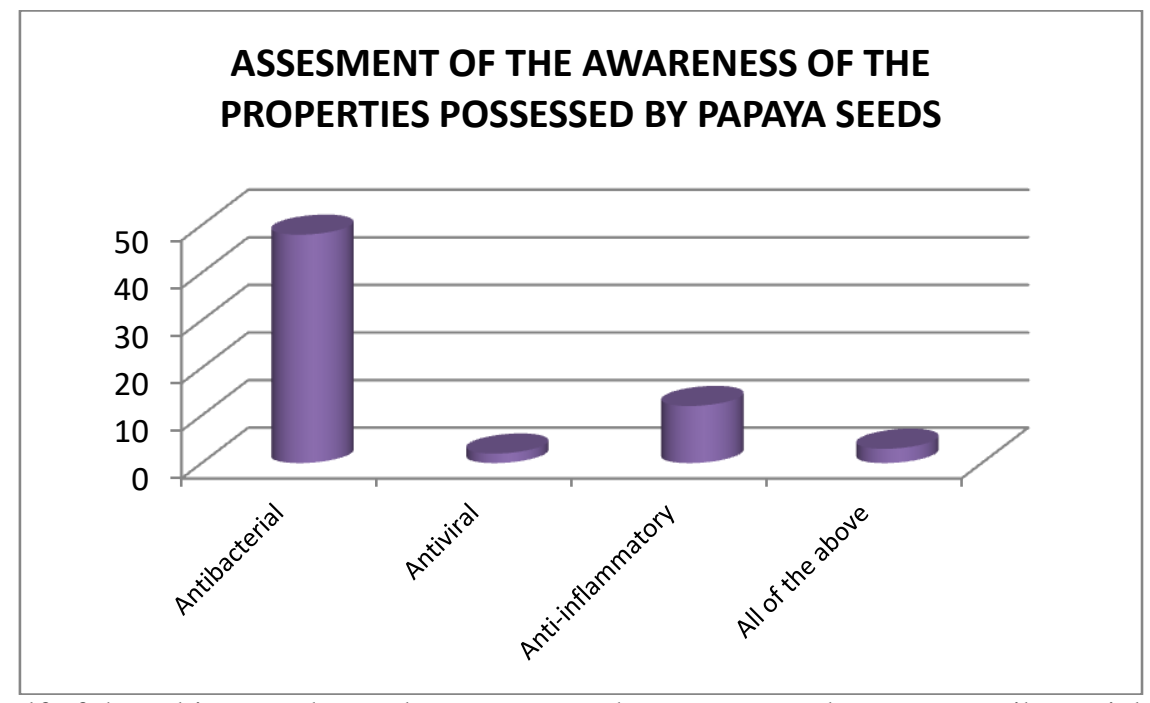

Almost half of the subjects under study were aware that papaya seeds possess antibacterial property.

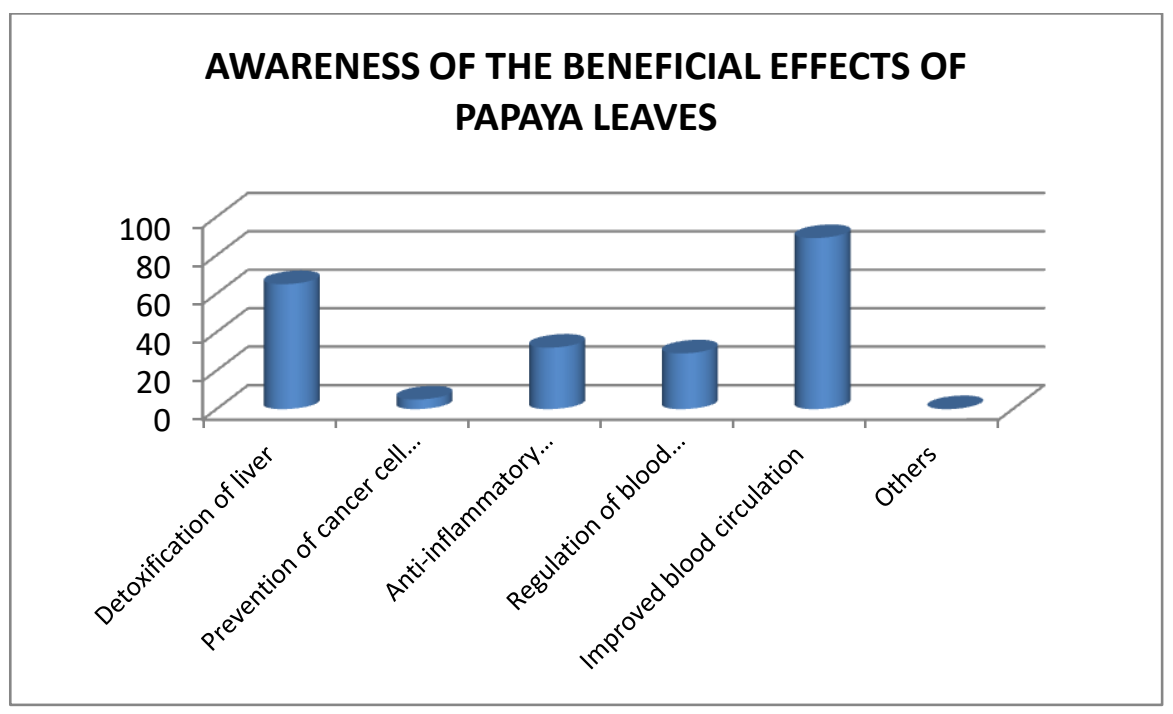

$89 \%$ of the subjects under study related the use of papaya in dengue treatment to improved blood circulation.

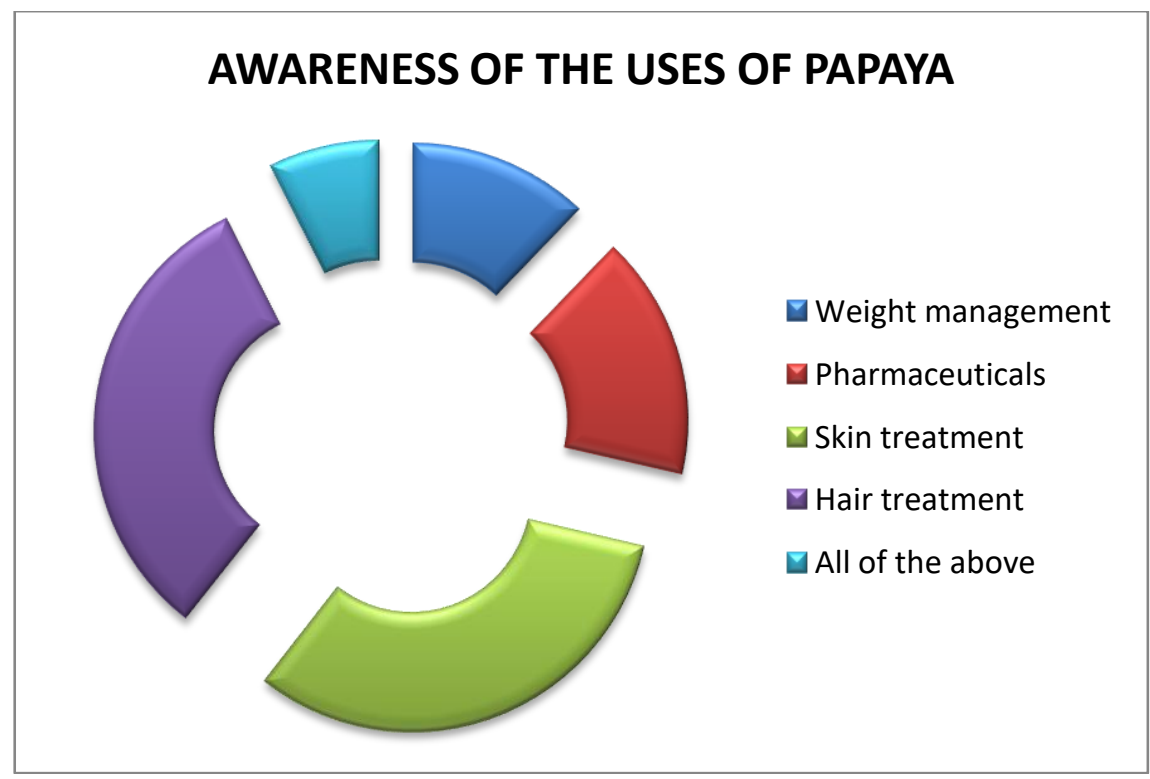

$95 \%$ of the subjects under study were aware of the usefulness of papaya in skin and hair treatment. 


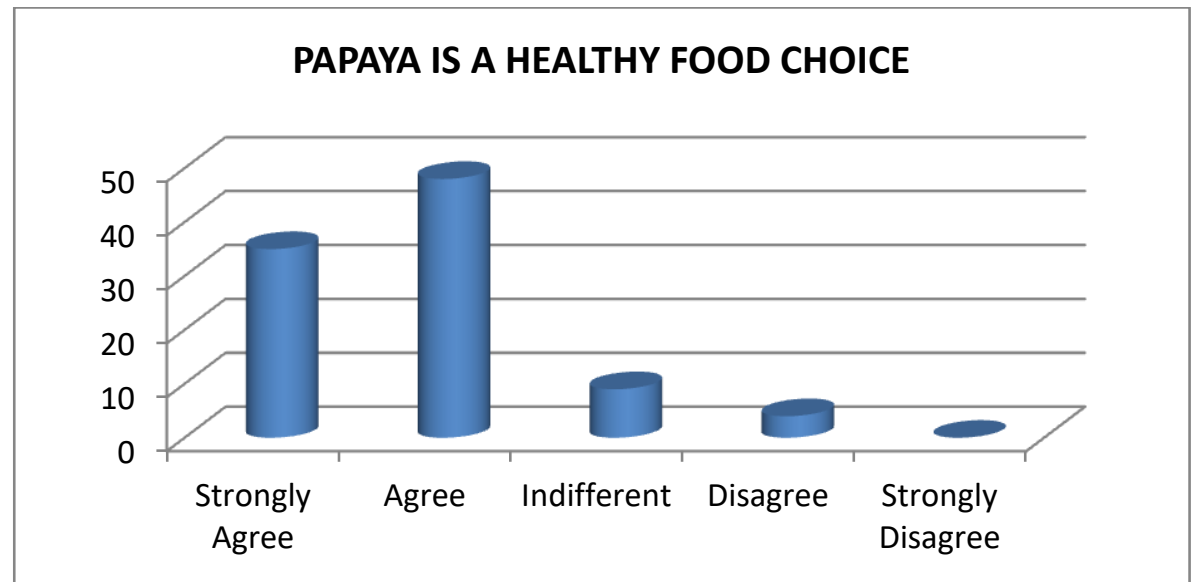

Most of the subjects agreed that papaya is a healthy food choice.

\section{CONCLUSION -}

There are many influences on food choice which provide a whole set of means to intervene into and improve people's food choices. There are also a number of barriers to dietary and lifestyle change, which vary depending on life stages and the individual or group of people in question.t is a major challenge both to health professionals and to the public themselves to effect dietary change. Different strategies are required to trigger a change in behaviour in groups with different priorities.The results are indicative of important benchmarks of psychosocial aspects of gastronomy, health management and consumerism. This research endeavour is relevant because it mirrors the 'correct' picture of use of specific traditional and superior nutrient choices in order to achieve sustainable health and wellness. However, the food choice, in relation to fruit intake, needs to be studied in more depth, in order to provide effective nutrition education programmes.

\section{REFERENCES -}

[1] Anderson A, Hetherington M, Adamson A, et al. (2003) The development and evaluation of a novel school based intervention to increase fruit and vegetable intake in children (Five a Day The Bash Street Way), N09003. Report for the FSA, London. (see http://www.food.gov.uk/)

[2] Clarke JE (1998) Taste and flavour: their importance in food choice and acceptance. Proceedings of the Nutrition Society 57: 639-643.

[3] Dibsdall LA, Lambert N, Bobbin RF, Frewer LJ (2003) Low-income consumers' attitudes and behaviour towards access, availability and motivation to eat fruit and vegetables. Public Health Nutrition 6(2):15968.

[4] Lassen A, Vibeke Thorsen A, Trolle E et al. (2004) Successful strategies to increase the consumption of fruits and vegetables: results from the Danish '6 a day' Worksite-Canteen Model Study. Public Health Nutrition 7(2): 263-70.

[5] Sorensen LB, Moller P, Flint A, Martens M, Raben A (2003). Effect of sensory perception of foods on appetite and food intake: a review of studies on humans. Int J Obes Relat Metab Disord. 27:1152-1166.

[6] Sorensen G, Stoddard A \& Macario E (1998b) Social support and readiness to make dietary changes. Health Education and Behaviour 25: 586-598.

[7] Stevens VJ , Glasgow RE, Toobert DJ, et al. (2002) Randomized trial of a brief dietary intervention to decrease consumption of fat and increase consumption of fruits and vegetables. American Journal of Health Promotion 16(3): 129-34.

[8] Wardle J, Steptoe A, Oliver G, Lipsey Z (2000) Stress, dietary restraint and food intake. Journal of Psychosomatic Research 48: 195-202. 